Bundesgesundheitsbl 2013 · 56:1003-1016 DOI 10.1007/s00103-013-1696-3

Online publiziert: 12. April 2013

๑) Springer-Verlag Berlin Heidelberg 2013
Bekanntmachung des Robert Koch-Instituts

\section{Falldefinitionen zur Übermittlung von Erkrankungs- und Todesfällen sowie von Erregernachweisen von Mumps, Pertussis, Röteln und Varizellen}

Die Liste der meldepflichtigen Krankheiten nach $\$ 6(1)$ Nr. 1 IfSG wurde mit Wirkung vom 29. März 2013 um Erkrankungs-, Verdachts- und Todesfälle von Mumps, Pertussis, Röteln und Varizellen erweitert. Gleichzeitig wurden der direkte oder indirekte Nachweis von Mumpsvirus, Bordetella pertussis, Bordetella parapertussis, Rötelnvirus und Varicella-Zoster-Virus in die Liste der meldepflichtigen Erregernachweise nach $\$ 7(1) \mathrm{Nr} .1$ IfSG aufgenommen. Die nichtnamentliche Meldung der konnatalen Röteln nach $\$ 7(3)$ entfällt [1].

Bislang waren diese Erkrankungen und Erreger nur durch landesspezifische Meldeverordnungen in den 5 östlichen Bundesländern meldepflichtig. Dazu waren mit den Landesstellen abgestimmte Falldefinitionen vom RKI erarbeitet und zuletzt im Epidemiologischen Bulletin publiziert worden [2].

Zukünftig werden nun bundesweit verlässliche Daten zur Krankheitslast dieser Krankheiten erhoben. Durch die namentliche Meldepflicht wird das Gesundheitsamt in die Lage versetzt, Ausbrüche dieser Erkrankungen frühzeitig zu erkennen und rechtzeitig Maßnahmen zur Verhinderung einer weiteren Ausbreitung beim Auftreten von Einzelfällen einzuleiten. Dies beinhaltet die Ermittlung von Kontaktpersonen, insbesondere solche mit einer besonderen Gefährdung, z. B. Schwangere (Röteln) oder Säuglinge (Pertussis, Varizellen) und die Durchführung von postexpositionellen Immunisierungen (bei Mumps, Röteln und Varizellen) oder einer Che- moprophylaxe (bei Pertussis). Gegen alle 4 Krankheiten stehen seit vielen Jahren wirksame Impfstoffe zur Verfügung und sind Impfungen bereits im Kindesalter allgemein empfohlen. Bundesweite Meldedaten zu den Erkrankungen können zukünftig auch Auskunft über die Wirksamkeit und Umsetzung der entsprechenden Impfempfehlungen auf Bevölkerungsebene geben und dazu beitragen, die Impfempfehlungen zu evaluieren und weiterzuentwickeln.

Aufgrund der einheitlichen bundesweiten Meldepflicht und vor dem Hintergrund geänderter diagnostischer Möglichkeiten sowie internationaler Erfordernisse der Krankheitsüberwachung wurden die Falldefinitionen zur Übermittlung von Erkrankungs- und Todesfällen sowie von Erregernachweisen von Mumps, Pertussis, Röteln und Varizellen vom RKI in Abstimmung mit den Landesstellen überarbeitet. Neu in der Struktur der Falldefinitionen ist die Unterscheidung zwischen dem spezifischen und dem unspezifischen klinischen Bild. Mit dieser Unterscheidung soll den Gesundheitsämtern die Kategorisierung der Fälle und die Entscheidung zur Fallübermittlung erleichtert und gleichzeitig die Bedeutung von Erregernachweisen unterstrichen werden. Bei Vorliegen eines entsprechenden Erregernachweises reicht auch ein unspezifisches klinisches Bild, um einen Fall als klinisch-labordiagnostisch oder klinisch-epidemiologisch $\mathrm{zu}$ klassifizieren und zu übermitteln.

Im Folgenden werden Aspekte der Meldepflicht der neu aufgenommenen
Krankheiten und Erreger erläutert und die Falldefinitionen bekannt gegeben.

\section{Mumps (s. Übersicht 1)}

Seit 1976 besteht in den westdeutschen Bundesländern eine Impfempfehlung der Ständigen Impfkommission (STIKO) gegen Mumps, die seitdem mehrmals verändert wurde. In den Neuen Bundesländern (NBL) wurde erst ab 1991 allgemein gegen Mumps geimpft. Seit 2001 besteht die aktuelle Empfehlung einer zweimaligen Impfung mit einem MMR-Kombinationsimpfstoff, wobei die 1. Dosis im Alter von 11 bis 14 Monaten und die 2. Dosis mit 15 bis 23 Monaten verabreicht werden soll.

Erkrankten vor Einführung der Mumps-Impfung Mitte der 70er-Jahre vorwiegend Kinder im Alter von 5 bis 9 Jahren, so kam es nach Einführung der Impfung aufgrund noch nicht ausreichender Impfquoten zu einer Verschiebung der Erkrankungen ins Jugend- und junge Erwachsenenalter. In den letzten Jahren ist es in Deutschland, aber auch in anderen Industrieländern zu teilweise großen und lang anhaltenden Mumps-Ausbrüchen in diesen Altersgruppen gekommen. Dabei fiel zusätzlich auf, dass auch vermehrt Erkrankungen bei 2-fach Geimpften auftraten, was als Indiz für eine über die Zeit nachlassende Wirkung des Impfstoffs (sog. „waning immunity“) angesehen werden kann. Daher gilt, dass auch eine 2-fache Impfung gegen Mumps bei entsprechen- 
den Symptomen eine Mumps-Erkrankung nicht ausschließt.

Mit einer bundesweiten Meldepflicht von Mumps sollen in Zukunft die häufiger auftretenden Ausbrüche frühzeitig erkannt werden, um rechtzeitig notwendige Schutzmaßnahmen, wie z. B. Riegelungsimpfungen, einzuleiten ( $\$ 16$ IfSG). Dies ist insbesondere im Hinblick auf die Rechtsverschiebung und häufig schwerer verlaufende Erkrankungen im höheren Alter wichtig, um so schwerwiegende Komplikationen, wie z. B. die Orchitis beim erwachsenen Mann, verhindern zu können.

Mithilfe der Meldedaten können bisher bestehende Impflücken aufgedeckt werden, und es kann versucht werden, diese zeitnah zu schließen. Außerdem können die Daten frühzeitig Hinweise auf mangelhaften oder nachlassenden Impfschutz geben und zur fundierten Begründung von Anpassungen der Impfempfehlungen beitragen $[3,4,5]$.

\section{Pertussis}

\section{(s. Übersicht 2)}

In der ehemaligen DDR bestand seit 1964 eine Impfpflicht gegen Pertussis. Dadurch wurde mit einer Inzidenz von $<1$ Erkrankung/100.000 Einwohner in den 1980er Jahren eine sehr niedrige Krankheitslast erreicht. In den alten Bundesländern (ABL) war die Impfung dagegen von 1969 bis 1974 empfohlen, dann aber bis auf wenige Ausnahmen für bestimmte RisikoKinder aufgrund z. T. unzureichend bestätigter Berichte schwerer Nebenwirkungen bis 1991 ausgesetzt [6]. Schätzungen der Krankheitslast in den 70er- und 80er-Jahren in den ABL lagen bei 160 bis 180 Erkrankungen/100.000 Einwohner. Nach der erneuten Empfehlung für eine Pertussis-Routineimpfung im Säuglingsund Kleinkindalter im Jahr 1991 stiegen die Impfquoten in den ABL nur langsam an, während sie in den NBL bedingt durch den Gesundheitssystemwechsel sogar vorübergehend abfielen, verbunden mit einem Anstieg der Erkrankungszahlen. Erst mit Einführung der azellulären Impfstoffe im Jahr 1995 fand ein stetiger Anstieg der Impfquoten in beiden Teilen Deutschlands statt, der aber wiederum schneller in den NBL voranschritt. So stiegen von 1998 bis 2010 die Impfquoten zum Zeitpunkt der Einschulung in den NBL von 85,8 auf $97,1 \%$ und in den ABL von 57,7 auf $94,3 \%$ an [7]. Daten des Kinder- und Jugendgesundheitssurveys aus den Jahren 2003 bis 2006 hatten zudem gezeigt, dass der Impfschutz insbesondere von Jugendlichen in den ABL noch große Lücken aufwies. So hatten nur $23,1 \%$ der 14- bis 17-jährigen Jugendlichen in den ABL und 78,8\% in den NBL eine Grundimmunisierung, und sogar nur 5,3\% (ABL) bzw. 39,5\% (NBL) hatten die aufgrund eines steigenden Anteils von Fällen bei Jugendlichen seit 2000 von der STIKO empfohlene Auffrischimpfung im Jugendalter erhalten [8].

Seit Einführung des IfSG im Jahr 2001 war Pertussis nur noch über Landesverordnungen in den NBL meldepflichtig. Nach diesen Daten stieg die Pertussis-Inzidenz trotz steigender Impfquoten dort von 2002 bis 2007 von 9,7 auf 39,8 Erkrankungen/100.000 Einwohner an, um dann bis 2010 auf 19,0 abzufallen. In den Jahren 2011 und 2012 wurde allerdings erneut ein Anstieg auf 32 bzw. 42 Erkrankungen/100.000 Einwohner verzeichnet. Den stärksten Anstieg zeigten diese Daten in den Jahren 2004 bis 2005 bei Kindern zwischen 5 und 14 Jahren, woraufhin von der STIKO 2006 eine Auffrischimpfung im Vorschulalter empfohlen wurde [9]. Nachfolgend konnte in den Jahren 2007 bis 2009 ein Rückgang der Pertussis-Fälle bei jüngeren Schulkindern in den NBL nachgewiesen werden [10]. Mit dem Anstieg der Fallzahlen in den Jahren 2011 bis 2012 stieg die Krankheitslast vor allem bei 10- bis 19-jährigen Kindern. Wegen des hohen Anteils der Erwachsenen unter allen gemeldeten Fällen in den NBL (75\% im Jahr 2008) wurde im Jahr 2009 eine Auffrischimpfung für Erwachsene bei der nächsten fälligen Auffrischung des Tetanus- und DiphtherieImpfschutzes eingeführt [11].

Internationale Experten sind sich einig, dass die Pertussis-Impfung weniger wirksam ist als andere Kinderimpfungen. Wie in einem jüngeren Leitartikel zusammengefasst [12], haben die derzeitigen azellulären Pertussis-Impfstoffe eine niedrigere Impfeffektivität als die meisten früheren Ganzkeimimpfstoffe. Nach Cherry [12] wurde die Effektivität der azellulären Impfstoffe in Studien überschätzt, weil häufig nur solche Kinder labordiagnostisch untersucht wurden, die eine sehr strenge klinische Falldefinition erfüllten. Zudem findet ein schneller Abfall der schützenden Antikörper statt, sodass regelmäßige Auffrischimpfungen voraussichtlich auch im Erwachsenenalter notwendig sein werden. Dagegen tragen genetische Veränderungen des Erregers bislang nicht zur verminderten Impfeffektivität bei. Dennoch sollte die Entwicklung neuer Impfstoffe vorangetrieben werden.

Vor diesem Hintergrund ist eine bundesweite Meldepflicht für Pertussis besonders wichtig. Die auf der Basis der Pertussis-Meldedaten aus den NBL berechnete Krankheitslast lässt sich nicht ohne weiteres auf die ABL übertragen. So ist z. B. die Hospitalisierungsrate für Pertussis bei Säuglingen in den ABL noch deutlich höher als in den NBL [10]. Auf die unterschiedliche Entwicklung der Impfquoten wurde bereits hingewiesen (s. oben). Mit der einheitlichen bundesweiten Meldepflicht sind die Gesundheitsämter auch berufen, Kontaktpersonen von an Pertussis erkrankten Patienten zu ermitteln um diesen eine Chemoprophylaxe zukommen zu lassen und damit weitere Infektionen - insbesondere bei ungeimpften Säuglingen - zu verhindern (\$16 IfSG) [13].

Die Falldefinitionen sollen den Gesundheitsämtern auch Hilfestellung bei den eigenen Ermittlungen bezüglich einer adäquaten Diagnostik und der Interpretation von Befunden geben. Das ist umso wichtiger, als die Diagnostik von Pertussis einige Besonderheiten aufweist:

Für die serologische Diagnostik sollte ein ELISA durchgeführt werden, der als Antigen ausschließlich Pertussis-Toxin (PT) enthält. ELISA-Testkits, die weitere Antigene enthalten, z. B. das filamentöse Hämagglutinin, können für andere Erreger kreuzreaktiv sein [14]. Zudem sollten vor allem IgG-Antikörpernachweise angestrebt werden, da IgM-Antikörper gegen Pertussis nicht aussagekräftig sind und IgA-Antikörper eine niedrige Sensitivität (aber hohe Spezifität) haben. Beim serologischen Nachweis sollte angestrebt werden, einen Antikörperanstieg nachzuweisen mittels einer zwei- 
ten Serumprobe 2 bis 4 Wochen nach der ersten, insbesondere wenn das IgG-antiPT zwischen 40 und 100 ELISA-Units/ $\mathrm{ml}(\mathrm{EU} / \mathrm{ml})$ liegt. Bei einem IgG-anti$\mathrm{PT}$ von $\geq 100 \mathrm{EU} / \mathrm{ml}$ ist bereits eine Serumprobe aussagekräftig. Werte $<40 \mathrm{EU} /$ $\mathrm{ml}$ schließen eine akute Erkrankung aus [15]. Andere serologische Methoden sind nicht zu empfehlen [14]. Die Mikroagglutination ist unzureichend spezifisch, Immunoblots sind nicht quantifizierbar. Wenn vor Kurzem gegen Pertussis geimpft wurde, ist die serologische Diagnostik nicht aussagekräftig, und es sollte eine PCR erfolgen [14]. Diesbezüglich wurde in der Falldefinition von 2009 noch ein Zeitraum 36 Monaten angegeben; dieser wurde jedoch basierend auf Expertenmeinungen [14] in der neuen bundesweit gültigen Falldefinition auf 12 Monate gekürzt. Bei der PCR ist zu beachten, dass eine Amplifikation von speziesspezifischen Gensequenzen (IS481 für B. pertussis, IS1001 für B. parapertussis) erfolgen sollte. In den USA wurde in einigen (Pseudo-)Ausbrüchen nachträglich ein hoher Anteil Parapertussis-Infektionen nachgewiesen [16].

\section{Röteln}

\section{(s. Übersicht 3)}

Die aufgrund der Meldedaten nach Landesverordnungen in den NBL berechnete Inzidenz von (postnatalen) Röteln lag seit 2007 fast durchweg bei weniger als 1 Fall pro 1 Mio. Einwohner. Im Jahr 2012 wurde allerdings eine Inzidenz von 2,5 Fällen pro 1 Mio. Einwohner errechnet. Von einer Unterschätzung aufgrund der unspezifischen Symptomatik der Röteln ist jedoch auszugehen. Aufgrund historisch unterschiedlicher Impfempfehlungen, Impfstoffverfügbarkeit und Impfinanspruchnahme in der früheren DDR und BRD bzw. den neuen und alten Bundesländern können auch die Röteln-Meldedaten der NBL nicht als repräsentativ für ganz Deutschland angesehen werden.

Während in Westdeutschland (BRD) bereits 1974 die Röteln-Impfung bei Mädchen eingeführt und 1980 dort als Kombinationsimpfung (mit Masern und Mumps) allen Kindern empfohlen wurde, war die Röteln-Impfung in der DDR nicht allgemein verfügbar. Seit 1991 wird die MMR-Impfung überall in Deutschland allen Kindern mit 2 Dosen empfohlen. Die aktuelle Impfempfehlung sieht die erste MMR-Impfung im Alter von 11 bis 14 Monaten und die zweite Dosis im Alter von 15 bis 23 Monaten vor. Darüber hinaus sollte darauf geachtet werden, dass alle Frauen im gebärfähigen Alter insgesamt 2 Impfungen gegen die Röteln in ihrem Leben erhalten haben.

Seit 2001 bestand nach Infektionsschutzgesetz $\$ 7(3)$ die nichtnamentliche Meldepflicht von konnatalen Röteln-Infektionen in Deutschland direkt an das Robert Koch-Institut [17]. Es wurden dem RKI pro Jahr nur sehr vereinzelt Fälle von konnatalen Röteln gemeldet. Auch hier ist von einer Unterschätzung der Fälle auszugehen, da z. B. rötelninduzierte Fehlgeburten, Abbrüche oder Totgeburten sowie konnatale Röteln, die erst später im Laufe des ersten Lebensjahres diagnostiziert werden, in der Regel nicht gemeldet wurden.

Die Elimination der Röteln und damit auch der konnatalen Röteln ist ein erklärtes Ziel der WHO, das bis 2015 erreicht werden soll. Auch Deutschland hat sich zu diesem Ziel verpflichtet. Für die Zertifizierung gilt es, den Nachweis zu erbringen, dass über einen Zeitraum von mindestens 12 Monaten keine endemischen Röteln-Fälle aufgetreten sind sowie Inzidenzen von weniger als 1 Röteln-Fall pro 1 Mio. Einwohner und weniger als 1 Fall von konnatalen Röteln auf 100.000 Lebendgeborene vorliegen [18]. Mit der einheitlichen bundesweiten Meldepflicht schafft Deutschland die für den Zertifizierungsprozess der WHO erforderlichen Voraussetzungen einer fallbasierten Surveillance und Inzidenzbestimmung auf Bevölkerungsebene.

Die Meldedaten werden dazu beitragen, die epidemiologische Lage in Deutschland korrekt bewerten zu können und das Risiko für eine Röteln-Infektion in besonderen Bevölkerungsgruppen wie Schwangeren ausreichend einschätzen zu können. Außerdem können die Meldedaten frühzeitig Hinweise auf eine sinkende Immunität in der Bevölkerung bzw. in bestimmten Bevölkerungsgruppen oder über einen vermehrten Import von Röteln aus dem Ausland geben.
Varizellen (s. Übersicht 4)

Die STIKO empfiehlt seit 2004 die Varizellen-Impfung allen Kindern, vorzugsweise im Alter von 11 bis 14 Monaten. Seit 2009 ist eine 2. Impfdosis vor vollendetem 2. Lebensjahr empfohlen. Darüber hinaus ist die Impfung allen ungeimpften Personen ohne Varizellen-Anamnese angeraten, die durch eine Varizellen-Infektion ein erhöhtes Risiko für einen komplizierten Verlauf der Erkrankung haben, sowie die postexpositionelle Impfung.

Die Meldepflicht für Varizellen wurde in den NBL nach Landesverordnungen $\mathrm{zu}$ unterschiedlichen Zeitpunkten zwischen 2002 und 2009 eingeführt. Daneben liegen aus dem Sentinel der Arbeitsgemeinschaft Varizellen seit April 2005 bundesweite Surveillancedaten vor, die im Gegensatz zu IfSG-Meldedaten jedoch keinen Bevölkerungsbezug erlauben. Die Sentineldaten zeigen einen deutlichen Rückgang der Erkrankungszahlen sowie der Häufigkeit von Komplikationen bei Varizellen seit Impfempfehlung [19, 20]. Bei zunehmenden Impfquoten und durch die allgemeine Empfehlung einer 2. Impfdosis werden die Erkrankungszahlen weiter zurückgehen. Damit ist ein Übergang von einer weitgehend endemischen Ausbreitung des Varizella-Zoster-Virus (VZV) hin zu räumlich und zeitlich beschränkten Ausbrüchen von Varizellen zu erwarten. Diese können durch Sentinelsysteme nicht mehr in jedem Fall erfasst werden, da der Ort des Ausbruchs nicht mit dem Standort der am Sentinel beteiligten Meldearztpraxen übereinstimmt. Andererseits würden ausbruchsbedingte Meldespitzen aus einzelnen Arztpraxen zu einer Verzerrung in der Einschätzung der Gesamtsituation führen. Bereits jetzt können mit dem Sentinelsystem selten auftretende Ereignisse, wie z. B. Komplikationen der Varizellen, nicht mehr zuverlässig erfasst werden.

Der Bevölkerungsbezug der Meldepflicht erlaubt die Berechnung altersspezifischer Inzidenzen. Diese sind eine wichtige Kenngröße dafür, ob es ggf. bei unzureichenden Impfquoten zu einer Verschiebung des Erkrankungsalters bei Varizellen in solche Altersgruppen kommt, die ein erhöhtes Risiko für Kom- 
plikationen bei Varizellen-Erkrankungen haben, wie Säuglinge oder Jugendliche und Erwachsene. Damit geben die Meldedaten Auskunft über die Auswirkungen der Varizellen-Impfung auf Bevölkerungsebene und sind eine wichtige Grundlage für evtl. notwendige Anpassungen der Impfempfehlung.

Das rechtzeitige Erkennen von und Reagieren auf Varizellen-Ausbrüche durch die Gesundheitsämter, das durch die Meldepflicht befördert wird, wird es auch ermöglichen, die Personen vor einer VZV-Infektion besser zu schützen, die selbst nicht geimpft werden können, jedoch ein erhöhtes Risiko für Komplikationen bei Varizellen-Erkrankung haben.

$\mathrm{Zu}$ beachten ist, dass der Erreger der Windpocken, das Varizella-Zoster-Virus, latent in den Nervenbahnen verbleibt und nach endogener Reaktivierung zum Krankheitsbild der Gürtelrose (Herpes zoster) führen kann. Während sich das klinische Bild der Gürtelrose von dem der Windpocken gut unterscheiden lässt, ist diese Unterscheidung beim Erregernachweis nicht möglich. Da der Labornachweis von Varicella-Zoster-Virus allein keine Unterscheidung zwischen Windpocken oder Herpes zoster zulässt, ist die Erhebung von klinischen Kriterien der Erkrankung besonders wichtig. Um den Unterschied zum klinischen Bild der Varizellen deutlich zu machen, ist in der Falldefinition (s. Übersicht 4; Meldepflicht nach $\$ \$ 6,7$ IfSG) darum auch das klinische Bild der Gürtelrose beschrieben. Darüber hinaus ist eine Meldepflicht des Herpes zoster nach Landesverordnungen in Brandenburg und Sachsen vorgesehen (Stand: September 2012).

Online publiziert: 12. April 2013 


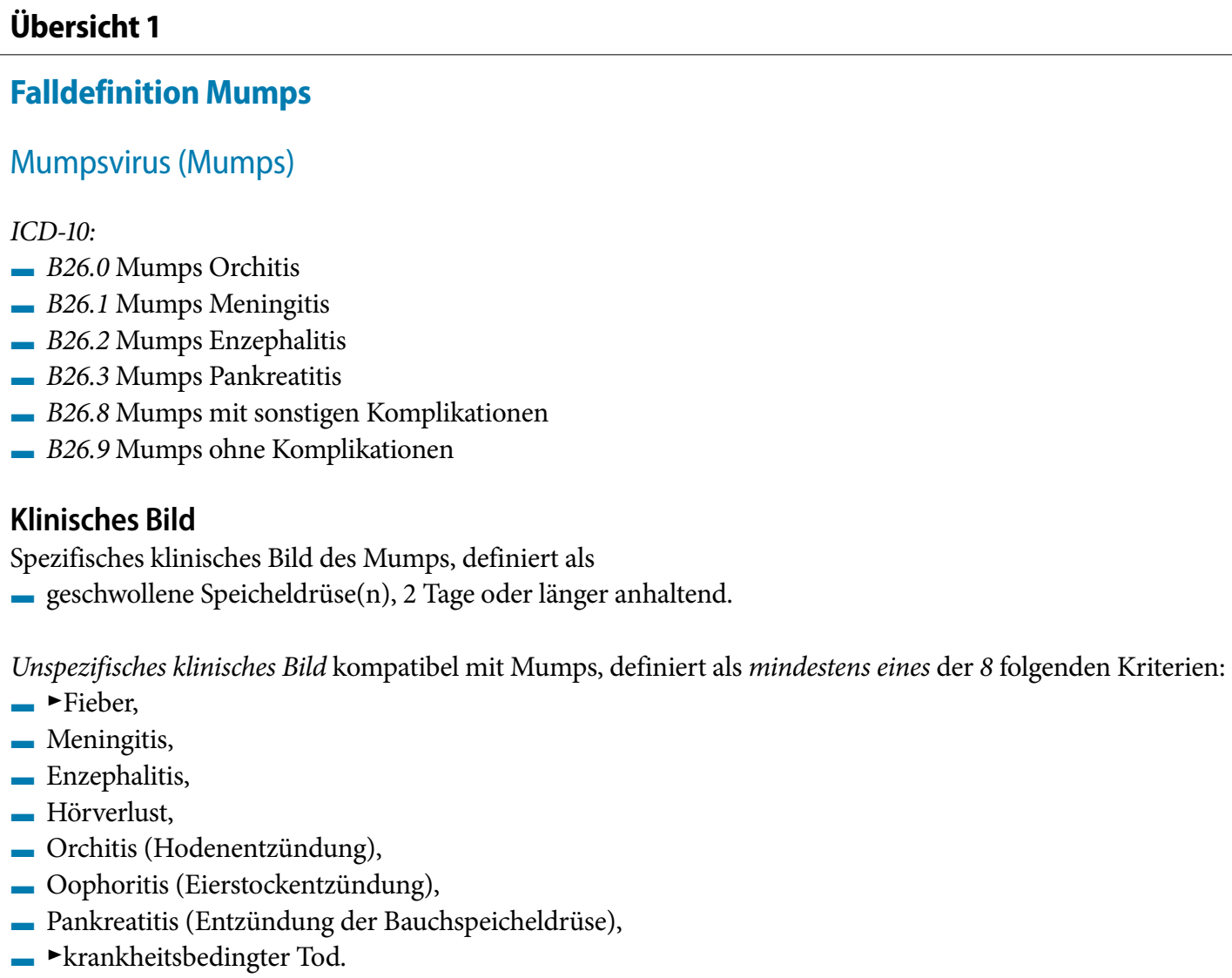

Zusatzinformation: Bei impfpräventablen Krankheiten sollten stets Angaben zur Impfanamnese (Anzahl der vorangegangenen Impfungen, Art und Datum der letzten Impfung) erhoben (z. B. Impfbuchkontrolle) und übermittelt werden.

\section{Labordiagnostischer Nachweis}

Positiver Befund mit mindestens einer der 5 folgenden Methoden:

[direkter Erregernachweis nur aus Rachenabstrich oder Rachenspülflüssigkeit, Speicheldrüsensekret, Zahntaschenflüssigkeit, Urin, Liquor, Biopsiematerial:]

- Erregerisolierung (kulturell),

- Nukleinsäurenachweis (z. B. PCR),

[indirekter (serologischer) Nachweis:]

- Nachweis Mumps-spezifischer IgM-Antikörper (z. B. ELISA),

- Nachweis Mumps-spezifischer IgG-Antikörper (z. B. ELISA) - deutliche Änderung zwischen 2 Proben,

- Nachweis intrathekal gebildeter Mumps-spezifischer Antikörper (erhöhter Liquor/Serum-Index).

Zusatzinformation: Die Bewertung von Virus- und Antikörpernachweisen setzt die Kenntnis eines evtl. zeitlichen Zusammenhangs mit einer Mumps-Impfung voraus. Darum sind beim Vorliegen labordiagnostischer Nachweise genaue Angaben zur letzten Impfung unerlässlich.

\section{Epidemiologische Bestätigung}

Epidemiologische Bestätigung, definiert als folgender Nachweis unter Berücksichtigung der Inkubationszeit:

- epidemiologischer Zusammenhang mit einer labordiagnostisch nachgewiesenen Infektion beim Menschen durch - Mensch-zu-Mensch-Übertragung.

Inkubationszeit ca. 16 bis 18 Tage (12 bis 25 Tage möglich). 


\section{Übersicht 1 (Fortsetzung)}

Über die zuständige Landesbehörde an das RKI zu übermittelnder Fall

A. Klinisch diagnostizierte Erkrankung

Spezifisches klinisches Bild des Mumps, ohne labordiagnostischen Nachweis und ohne epidemiologische Bestätigung.

B. Klinisch-epidemiologisch bestätigte Erkrankung

Spezifisches oder unspezifisches klinisches Bild kompatibel mit Mumps ohne labordiagnostischen Nachweis, aber mit epidemiolo-

gischer Bestätigung.

C. Klinisch-labordiagnostisch bestätigte Erkrankung

Spezifisches oder unspezifisches klinisches Bild kompatibel mit Mumps und labordiagnostischer Nachweis.

D. Labordiagnostisch nachgewiesene Infektion bei nicht erfülltem klinischem Bild

Labordiagnostischer Nachweis bei bekanntem klinischem Bild, das weder die Kriterien für das spezifische noch das unspezifische klinische Bild des Mumps erfüllt. Hierunter fallen auch asymptomatische Infektionen.

E. Labordiagnostisch nachgewiesene Infektion bei unbekanntem klinischem Bild

Labordiagnostischer Nachweis bei fehlenden Angaben zum klinischen Bild (nicht ermittelbar oder nicht erhoben).

\section{Referenzdefinition}

In Veröffentlichungen des Robert Koch-Instituts, die nicht nach Falldefinitionskategorien differenzieren, werden nur Erkrankungen der Kategorien $A, B$ und $C$ gezählt.

\section{Gesetzliche Grundlage}

Meldepflicht: Dem Gesundheitsamt werden gemäß $₫ 6$ Abs. 1, Satz 1, Nr. 1 Buchst. k IfSG der Krankheitsverdacht, die Erkrankung sowie der Tod an Mumps sowie gemäß $\$ 7$ Abs. 1 Nr. 32 IfSG der direkte oder indirekte Nachweis des Mumpsvirus, soweit er auf eine akute Infektion hinweist, namentlich gemeldet. Darüber hinaus stellt das Gesundheitsamt gemäß $\S 25$ Abs. 1 IfSG ggf. eigene Ermittlungen an.

Übermittlung: Das Gesundheitsamt übermittelt gemäß $\$ 11$ Abs. 1 IfSG an die zuständige Landesbehörde nur Erkrankungs- oder Todesfälle und Erregernachweise, die der Falldefinition gemäß $₫ 4$ Abs. 2 Nr. 2 Buchst. a IfSG entsprechen. 


\section{Übersicht 2 \\ Falldefinition Pertussis \\ Bordetella pertussis und Bordetella parapertussis (Keuchhusten) \\ ICD10: \\ - A37. Keuchhusten \\ - A37.0 Keuchhusten durch Bordetella pertussis \\ - A37.1 Keuchhusten durch Bordetella parapertussis \\ - A37.8 Keuchhusten durch sonstige Bordetella-Spezies \\ - A37.9 Keuchhusten, nicht näher bezeichnet}

\section{Klinisches Bild}

Klinisches Bild eines Keuchhustens, definiert entsprechend den folgenden Altersgruppen:

Bei Personen im Alter von $\geq 1$ Jahr:

- Mindestens eines der 2 folgenden Kriterien:

- mindestens 14 Tage anhaltender Husten UND mindestens eines der 3 folgenden Kriterien:

- anfallsweise auftretender Husten,

- inspiratorischer Stridor,

- Erbrechen nach den Hustenanfällen;

- - krankheitsbedingter Tod.

Bei Kindern im ersten Lebensjahr:

Mindestens eines der 2 folgenden Kriterien:

- Husten UND Apnoen,

- krankheitsbedingter Tod.

Zusatzinformationen: Bei impfpräventablen Krankheiten sollten stets Angaben zur Impfanamnese (Anzahl der vorangegangenen Impfungen, Art und Datum der letzten Impfung) erhoben (z. B. Impfbuchkontrolle) und übermittelt werden.

\section{Labordiagnostischer Nachweis}

Positiver Befund mit mindestens einer der 4 folgenden Methoden:

[direkter Erregernachweis nur aus Abstrichen oder Sekreten des Nasen-Rachen-Raums:]

- Isolierung von B. pertussis oder B. parapertussis (kulturell),

- Nukleinsäurenachweis (z. B. PCR) von Gensequenzen spezifisch für B. pertussis oder B. parapertussis,

[indirekter (serologischer) Nachweis:]

- Nachweis IgG- oder IgA-Antikörper gegen das Pertussis-Toxin (z. B. ELISA) - einmalig deutlich erhöhter Wert,

- Nachweis IgG- oder IgA-Antikörper gegen das Pertussis-Toxin • deutliche Änderung zwischen 2 Proben.

Zusatzinformation: Die Bewertung von Antikörpernachweisen setzt die Kenntnis eines evtl. zeitlichen Zusammenhangs mit einer Pertussis-Impfung voraus. Darum sind beim Vorliegen labordiagnostischer Nachweise genaue Angaben zur letzten Impfung unerlässlich.

\section{Epidemiologische Bestätigung}

Epidemiologische Bestätigung, definiert als folgender Nachweis unter Berücksichtigung der Inkubationszeit:

- epidemiologischer Zusammenhang mit einer labordiagnostisch nachgewiesenen Infektion beim Menschen durch

- Mensch-zu-Mensch-Übertragung.

Inkubationszeit ca. 9 bis 10 Tage (6 bis 20 Tage möglich). 


\section{Übersicht 2 (Fortsetzung)}

Über die zuständige Landesbehörde an das RKI zu übermittelnder Fall

A. Klinisch diagnostizierte Erkrankung

Entfällt.

B. Klinisch-epidemiologisch bestätigte Erkrankung

Klinisches Bild eines Keuchhustens ohne labordiagnostischen Nachweis, aber mit epidemiologischer Bestätigung.

C. Klinisch-labordiagnostisch bestätigte Erkrankung

Klinisches Bild eines Keuchhustens und labordiagnostischer Nachweis.

D. Labordiagnostisch nachgewiesene Infektion bei nicht erfülltem klinischem Bild

Labordiagnostischer Nachweis bei bekanntem klinischem Bild, das die Kriterien für einen Keuchhusten nicht erfüllt.

E. Labordiagnostisch nachgewiesene Infektion bei unbekanntem klinischem Bild

Labordiagnostischer Nachweis bei fehlenden Angaben zum klinischen Bild (nicht ermittelbar oder nicht erhoben).

Referenzdefinition

In Veröffentlichungen des Robert Koch-Instituts, die nicht nach Falldefinitionskategorien differenzieren, werden nur Erkrankungen der Kategorien $B$ und $C$ gezählt.

\section{Gesetzliche Grundlage}

Meldepflicht: Dem Gesundheitsamt werden gemäß $\$ 6$ Abs. 1, Satz 1, Nr. 1 Buchst. 1 IfSG der Krankheitsverdacht, die Erkrankung sowie der Tod an Pertussis durch Bordetella pertussis oder Bordetella parapertussis, sowie gemäß $\$ 7$ Abs. 1 Nr. 3 IfSG der direkte oder indirekte Nachweis von Bordetella pertussis oder Bordetella parapertussis, soweit er auf eine akute Infektion hinweist, namentlich gemeldet. Darüber hinaus stellt das Gesundheitsamt gemäß $₫ 25$ Abs. 1 IfSG ggf. eigene Ermittlungen an.

Übermittlung: Das Gesundheitsamt übermittelt gemäß $₫ 11$ Abs. 1 IfSG an die zuständige Landesbehörde nur Erkrankungs- oder Todesfälle und Erregernachweise, die der Falldefinition gemäß $₫ 4$ Abs. 2 Nr. 2 Buchst. a IfSG entsprechen. 


\section{Übersicht 3 \\ Röteln und konnatale Röteln \\ A. Rötelnviren (Röteln, postnatale Infektion) \\ ICD10: \\ - B06 Röteln \\ - B06.0 Röteln mit neurologischen Komplikationen \\ - B06.8 Röteln mit sonstigen Komplikationen \\ - B06.9 Röteln ohne Komplikationen \\ - M01.4 Arthritis bei Röteln \\ - J17.1 Pneumonie bei Röteln}

\section{Klinisches Bild}

Klinisches Bild der Röteln, definiert als mindestens eines der 2 folgenden Kriterien

- generalisierter Ausschlag (makulopapulös) UND mindestens eines der 2 folgenden Kriterien:

- Lymphadenopathie im Kopf-, Hals- oder Nackenbereich,

- Arthritis/Arthralgien;

- - krankheitsbedingter Tod.

Zusatzinformation: Bei impfpräventablen Krankheiten sollten stets Angaben zur Impfanamnese (Anzahl der vorangegangenen Impfungen, Art und Datum der letzten Impfung) erhoben (z. B. Impfbuchkontrolle) und übermittelt werden.

\section{Labordiagnostischer Nachweis}

Positiver Befund mit mindestens einer der 5 folgenden Methoden:

[direkter Erregernachweis nur aus Rachenabstrich oder Rachenspülflüssigkeit, Blut, Zahntaschenflüssigkeit, Urin, Liquor:]

- Erregerisolierung (kulturell),

- Nukleinsäurenachweis (z. B. PCR),

[indirekter (serologischer) Nachweis:]

- Nachweis Röteln-spezifischer IgM-Antikörper (z. B. ELISA),

- Nachweis Röteln-spezifischer IgG-Antikörper (z. B. ELISA) -deutliche Änderung zwischen 2 Proben,

- Nachweis intrathekal gebildeter Röteln-spezifischer Antikörper (erhöhter Liquor/Serum-Index).

Zusatzinformation: Die Bewertung von Virus- und Antikörpernachweisen setzt die Kenntnis eines evtl. zeitlichen Zusammenhangs mit einer Röteln-Impfung voraus. Darum sind beim Vorliegen labordiagnostischer Nachweise genaue Angaben zur letzten Impfung unerlässlich.

Ein Nachweis Röteln-spezifischer IgM-Antikörper in der Schwangerschaft bedarf vor therapeutischen Entscheidungen unbedingt einer weiteren Bestätigung durch eine Feindiagnostik (z. B. Western Blot, IgG-Avidität, direkter Erregernachweis).

\section{Epidemiologische Bestätigung}

Epidemiologische Bestätigung, definiert als folgender Nachweis unter Berücksichtigung der Inkubationszeit:

- epidemiologischer Zusammenhang mit einer labordiagnostisch nachgewiesenen Infektion beim Menschen

- durch Mensch-zu-Mensch-Übertragung.

Inkubationszeit ca. 14 bis 21 Tage.

\section{Über die zuständige Landesbehörde an das RKI zu übermittelnder Fall}

A. Klinisch diagnostizierte Erkrankung

Klinisches Bild der Röteln, ohne labordiagnostischen Nachweis und ohne epidemiologische Bestätigung.

B. Klinisch-epidemiologisch bestätigte Erkrankung

Klinisches Bild der Röteln, ohne labordiagnostischen Nachweis aber mit epidemiologischer Bestätigung.

C. Klinisch-labordiagnostisch bestätigte Erkrankung

Klinisches Bild der Röteln und labordiagnostischer Nachweis. 


\section{Übersicht 3 (Fortsetzung)}

\section{Labordiagnostisch nachgewiesene Infektion bei nicht erfülltem klinischem Bild}

Labordiagnostischer Nachweis bei bekanntem klinischem Bild, das die Kriterien für Röteln nicht erfüllt. Hierunter fallen auch asymptomatische Infektionen.

\section{E. Labordiagnostisch nachgewiesene Infektion bei unbekanntem klinischem Bild}

Labordiagnostischer Nachweis bei fehlenden Angaben zum klinischen Bild (nicht ermittelbar oder nicht erhoben).

\section{Referenzdefinition}

In Veröffentlichungen des Robert Koch-Instituts, die nicht nach Falldefinitionskategorien differenzieren, werden nur Erkrankungen der Kategorien $B$ und $C$ gezählt.

\section{Gesetzliche Grundlage}

Meldepflicht: Dem Gesundheitsamt werden gemäß $₫ 6$ Abs. 1, Satz 1, Nr. 1 Buchst. o IfSG der Krankheitsverdacht, die Erkrankung sowie der Tod an Röteln, sowie gemäß $\$ 7$ Abs. 1 Nr. 41 IfSG der direkte oder indirekte Nachweis von Röteln-Viren, soweit er auf eine akute Infektion hinweist, namentlich gemeldet. Darüber hinaus stellt das Gesundheitsamt gemäß $\$ 25$ Abs. 1 IfSG ggf. eigene Ermittlungen an.

Übermittlung: Das Gesundheitsamt übermittelt gemäß $\$ 11$ Abs. 1 IfSG an die zuständige Landesbehörde nur Erkrankungs- oder Todesfälle und Erregernachweise, die der Falldefinition gemäß $\$ 4$ Abs. 2 Nr. 2 Buchst. a IfSG entsprechen.

\section{B. Konnatale/kongenitale Röteln (Röteln-Embryofetopathie, konnatale Röteln-Infektion)}

ICD10:

- P35.0 Röteln-Embryopathie

- Kongenitale Röteln-Pneumonie

\section{Klinisches Bild}

Spezifisches klinisches Bild einer Röteln-Embryofetopathie, definiert als ein Kriterium der Kategorie A sowie mindestens ein weiteres Kriterium der Kategorie A oder B bei einem Kind im ersten Lebensjahr oder einer Totgeburt.

Unspezifisches klinisches Bild kompatibel mit einer Röteln-Embryofetopathie, definiert als ein Kriterium der Kategorie A bei einem Kind im ersten Lebensjahr oder einer Totgeburt oder $\bullet$ krankheitsbedingter Tod im ersten Lebensjahr.

Kategorie A (Leitsymptome)

- Innenohrtaubheit (uni- oder bilateral),

- kongenitaler Herzfehler (z. B. Septumdefekt, Ductus arteriosus, periphere Stenose der Arteria pulmonalis),

- Retinopathie,

- Katarakt,

- Glaukom.

Kategorie B (weitere Symptome)

- Splenomegalie,

- Mikrozephalus,

- Purpura,

- Entwicklungsverzögerung,

- Meningoenzephalitis,

- Ikterus mit Beginn innerhalb der ersten 24 h nach Geburt,

- Atrophie der Knochen mit vermehrter Strahlendurchlässigkeit.

Zusatzinformation: Bei impfpräventablen Krankheiten sollten stets Angaben zur Impfanamnese (Anzahl der vorangegangenen Impfungen, Art und Datum der letzten Impfung) von Mutter und ggf. Kind erhoben (z. B. Impfbuchkontrolle) und übermittelt werden.

\section{Labordiagnostischer Nachweis}

Positiver Befund mit mindestens einer der 5 folgenden Methoden:

[direkter Erregernachweis nur aus Material vom Kind oder der Plazenta]:

- Erregerisolierung (kulturell),

- Nukleinsäurenachweis (z. B. PCR), 


\section{Übersicht 3 (Fortsetzung)}

[indirekter (serologischer) Nachweis nur aus Material vom Kind:]

- Nachweis Röteln-spezifischer IgM-Antikörper (z. B. ELISA),

- mindestens zweimaliger fortbestehender Nachweis Röteln-spezifischer IgG-Antikörper zwischen dem 6. und 12. Lebensmonat, idealerweise im Abstand von 3 Monaten (z. B. bei der U5 und der U6),

- Nachweis intrathekal gebildeter Röteln-spezifischer Antikörper (erhöhter Liquor/Serum-Index).

Zusatzinformation: Eine labordiagnostische Untersuchung sollte so früh wie möglich nach der Geburt erfolgen. Bei der Interpretation labordiagnostischer Befunde (insbesondere, wenn diese erst spät im 1. Lebensjahr erhoben werden) muss die RötelnImpfanamnese sowie - z. B. bei einer Ausbruchssituation - auch die Möglichkeit einer postnatalen Röteln-Infektion des Kindes mit berücksichtigt werden. Kinder mit konnataler Röteln-Embryofetopathie können das Virus noch Monate nach der Geburt ausscheiden.

\section{Epidemiologische Bestätigung}

Epidemiologischer Zusammenhang mit einer labordiagnostisch nachgewiesenen akuten Infektion bei der Mutter während der Schwangerschaft.

\section{Über die zuständige Landesbehörde an das RKI zu übermittelnder Fall}

A. Klinisch diagnostizierte Erkrankung

Spezifisches klinisches Bild einer Röteln-Embryofetopathie, ohne labordiagnostischen Nachweis und ohne epidemiologische Bestätigung.

\section{B. Klinisch-epidemiologisch bestätigte Erkrankung}

Spezifisches oder unspezifisches klinisches Bild kompatibel mit einer Röteln-Embryofetopathie, ohne labordiagnostischen Nachweis, aber mit epidemiologischer Bestätigung.

\section{Klinisch-labordiagnostisch bestätigte Erkrankung}

Spezifisches oder unspezifisches klinisches Bild kompatibel mit einer Röteln-Embryofetopathie und labordiagnostischer Nachweis. D. Labordiagnostisch nachgewiesene Infektion bei nicht erfülltem klinischem Bild (konnatale Infektion)

Labordiagnostischer Nachweis bei bekanntem klinischem Bild, das weder die Kriterien für das spezifische noch das unspezifische klinische Bild einer Röteln-Embryofetopathie erfüllt. Hierunter fallen auch asymptomatische Infektionen.

E. Labordiagnostisch nachgewiesene Infektion bei unbekanntem klinischem Bild

Labordiagnostischer Nachweis bei fehlenden Angaben zum klinischen Bild (nicht ermittelbar oder nicht erhoben).

\section{Referenzdefinition}

In Veröffentlichungen des Robert Koch-Instituts, die nicht nach Falldefinitionskategorien differenzieren, werden nur Erkrankungen der Kategorien $A, B$ und $C$ gezählt.

\section{Gesetzliche Grundlage}

Meldepflicht: Dem Gesundheitsamt werden gemäß $₫ 6$ Abs. 1, Satz 1, Nr. 1 Buchst. o IfSG der Krankheitsverdacht, die Erkrankung sowie der Tod an Röteln sowie gemäß $\$ 7$ Abs. 1 Nr. 41 IfSG der direkte oder indirekte Nachweis von Röteln-Viren, soweit er auf eine akute Infektion hinweist, namentlich gemeldet. Darüber hinaus stellt das Gesundheitsamt gemäß $\$ 25$ Abs. 1 IfSG ggf. eigene Ermittlungen an.

Übermittlung: Das Gesundheitsamt übermittelt gemäß $\$ 11$ Abs. 1 IfSG an die zuständige Landesbehörde nur Erkrankungs- oder Todesfälle und Erregernachweise, die der Falldefinition gemäß $₫ 4$ Abs. 2 Nr. 2 Buchst. a IfSG entsprechen. 


\section{Übersicht 4}

Varizellen

Varicella-Zoster-Virus (VZV - Windpocken und Herpes zoster)

ICD10:

- B01 Varizellen [Windpocken]

- B01.0 Varizellen-Meningitis

- B01.1 Varizellen-Enzephalitis

- B01.2 Varizellen-Pneumonie

- B01.8 Varizellen mit sonstigen Komplikationen

- B01.9 Varizellen ohne Komplikationen

- B02 Zoster [Herpes zoster], inklusive Gürtelrose, Herpes zoster

- B02.0 Zoster-Enzephalitis (G05.1*), Zoster-Meningoenzephalitis

- B02.1 Zoster-Meningitis (G02.0*)

- B02.2 Zoster mit Beteiligung anderer Abschnitte des Nervensystems

- B02.3 Zoster ophthalmicus

- B02.7 Zoster generalisatus

- B02.8 Zoster mit sonstigen Komplikationen

- B02.9 Zoster ohne Komplikationen

\section{Klinisches Bild}

Varicella-Zoster-Erkrankung, definiert als mindestens eine der 2 folgenden Formen:

Windpocken:

Spezifisches klinisches Bild der Windpocken, definiert als:

- anfangs makulopapulöser Ausschlag an Haut oder Schleimhaut bei nachfolgend gleichzeitig vorhandenen Papeln, Bläschen bzw. Pusteln und Schorf (sog. Sternenhimmel).

Unspezifisches klinisches Bild kompatibel mit Windpocken, definiert als mindestens eines der 2 folgenden Kriterien:

- Ausschlag an Haut oder Schleimhaut mit Flecken, Bläschen oder Pusteln,

- $>$ krankheitsbedingter Tod.

Herpes zoster':

Spezifisches klinisches Bild des Herpes zoster, definiert als:

— ein meist einseitig auf ein Hautsegment (Dermatom) beschränkter, bläschenförmiger Ausschlag

UND mindestens eines der 4 folgenden Kriterien:

- Schmerzen im betroffenen Bereich („Zosterneuralgie“),

- Fieber,

- Gliederschmerzen,

- Brennen oder Juckreiz am Ort des Auftretens.

Unspezifisches klinisches Bild kompatibel mit Herpes zoster, definiert als mindestens eines der 2 folgenden Kriterien: - einseitig auf ein Hautsegment lokalisierte Schmerzen ohne bläschenförmigen Ausschlag (Zoster sine herpete),

- $\bullet$ krankheitsbedingter Tod.

Zusatzinformation: Bei impfpräventablen Krankheiten sollten stets Angaben zur Impfanamnese (Anzahl der vorangegangenen Impfungen, Art und Datum der letzten Impfung) erhoben (z. B. Impfbuchkontrolle) und übermittelt werden.

\section{Labordiagnostischer Nachweis}

Positiver Befund mit mindestens einer der 6 folgenden Methoden:

[direkter Erregernachweis nur aus Bläscheninhalt, Liquor, bronchoalveolärer Lavage (BAL), Blut, Fruchtwasser oder Gewebe:]

- Erregerisolierung (kulturell),

- Nukleinsäurenachweis (z. B. PCR),

- Antigennachweis (z. B. IFT), 


\section{Übersicht 4 (Fortsetzung)}

[indirekter (serologischer) Nachweis:]

- Nachweis VZV-spezifischer IgM-Antikörper (z. B. ELISA),

- -deutliche Änderung zwischen 2 Proben beim VZV-spezifischen IgG- oder IgA-Antikörpernachweis (z. B. ELISA),

- Nachweis intrathekal gebildeter VZV-spezifischer Antikörper (erhöhter Liquor/Serum-Index).

Zusatzinformation: Die Bewertung von Virus- und Antikörpernachweisen setzt die Kenntnis eines evtl. zeitlichen Zusammenhangs mit einer Windpocken- oder Herpes-Zoster-Impfung voraus. Darum sind beim Vorliegen labordiagnostischer Nachweise genaue Angaben zur letzten Impfung unerlässlich.

\section{Epidemiologische Bestätigung}

Epidemiologische Bestätigung, definiert als folgender Nachweis unter Berücksichtigung der Inkubationszeit:

- epidemiologischer Zusammenhang mit einer labordiagnostisch nachgewiesenen Infektion beim Menschen

- durch Mensch-zu-Mensch-Übertragung.

Inkubationszeit ca. 8 bis 28 Tage.

\section{Über die zuständige Landesbehörde an das RKI zu übermittelnder Fall}

A. Klinisch diagnostizierte Erkrankung

Spezifisches klinisches Bild der Windpocken ohne labordiagnostischen Nachweis und ohne epidemiologische Bestätigung.

B. Klinisch-epidemiologisch bestätigte Erkrankung

Spezifisches oder unspezifisches klinisches Bild kompatibel mit Windpocken, ohne labordiagnostischen Nachweis, aber mit epidemiologischer Bestätigung.

C. Klinisch-labordiagnostisch bestätigte Erkrankung

Spezifisches oder unspezifisches klinisches Bild kompatibel mit Windpocken oder Herpes zoster und labordiagnostischer Nachweis.

D. Labordiagnostisch nachgewiesene Infektion bei nicht erfülltem klinischem Bild

Labordiagnostischer Nachweis bei bekanntem klinischem Bild, das weder die Kriterien für das spezifische noch das unspezifische klinische Bild der Windpocken bzw. des Herpes zoster erfüllt. Hierunter fallen auch asymptomatische Infektionen.

E. Labordiagnostisch nachgewiesene Infektion bei unbekanntem klinischem Bild

Labordiagnostischer Nachweis bei fehlenden Angaben zum klinischen Bild (nicht ermittelbar oder nicht erhoben).

\section{Referenzdefinition}

In Veröffentlichungen des Robert Koch-Instituts, die nicht nach Falldefinitionskategorien differenzieren (z. B. wöchentliche „Aktuelle Statistik meldepflichtiger Infektionskrankheiten“ im Epidemiologischen Bulletin), werden nur Erkrankungen an Windpocken der Kategorien $A, B$ und $C$ gezählt.

\section{Gesetzliche Grundlage}

Meldepflicht: Dem Gesundheitsamt werden gemäß $\$ 6$ Abs. 1, Satz 1, Nr. 1 Buchst. r IfSG der Krankheitsverdacht, die Erkrankung sowie der Tod an Windpocken sowie gemäß $₫ 7$ Abs. 1 Nr. 47 IfSG der direkte oder indirekte serologische Nachweis von Varicella-

Zoster-Virus, soweit er auf eine akute Infektion hinweist, namentlich gemeldet. Darüber hinaus stellt das Gesundheitsamt nach $₫ 25$ Abs. 1 IfSG ggf. eigene Ermittlungen an.

Übermittlung: Das Gesundheitsamt übermittelt gemäß $\$ 11$ Abs. 1 IfSG an die zuständige Landesbehörde nur Erkrankungs- oder Todesfälle und Erregernachweise, die der Falldefinition gemäß $₫ 4$ Abs. 2 Nr. 2 Buchst. A IfSG entsprechen.

\footnotetext{
${ }^{1}$ Herpes zoster ist eine Reaktivierung von Varicella-Zoster-Viren, die nach Erstinfektion, die sich klinisch als Windpocken zeigt, in Nervenwurzeln des Rückenmarks oder Gehirns verbleiben. Da der Labornachweis von Varicella-Zoster-Virus allein keine Unterscheidung zwischen Windpocken oder Herpes zoster zulässt, ist die Erhebung von klinischen Kriterien der Erkrankung besonders wichtig. Darüber hinaus ist eine Erkrankung an Herpes zoster in einigen Bundesländern aufgrund von Landesverordnungen meldepflichtig.
} 


\section{Literatur}

1. Gesetz zur Durchführung der Internationalen Gesundheitsvorschriften und zur Änderung weiterer Gesetze. Veröff. im Bundesanzeiger am 28.03.2013. http://www.bgbl.de/Xaver/start. xav?startbk=Bundesanzeiger_BGBI

2. Robert Koch-Institut (2009) Krankheiten, für die gemäß LVO eine erweiterte Meldepflicht zusätzlich zum IfSG besteht (Stand 2009). Epidemiol Bull 5:33-49

3. Anis E, Grotto I, Moerman L et al (2012) Mumps outbreak in Israel's highly vaccinated society: Are two doses enough? Epidemiol Infect 140(3):439446

4. Date AA, Kyaw MH, Rue AM et al (2008) Longterm persistence of mumps antibody after receipt of 2 measles-mumps-rubella (MMR) vaccinations and antibody response after a third MMR vaccination among a university population. J Infect Dis 197(12):1662-1668

5. Dayan GH, Quinlisk MP, Parker AA et al (2008) Recent resurgence of mumps in the United States. N Engl J Med 358(15):1580-1589

6. Hellenbrand W, Beier D, Jensen E et al (2009) The epidemiology of pertussis in Germany: past and present. BMC Infect Dis 9(22). http://www.biomedcentral.com/1471-2334/9/22

7. Robert Koch-Institut (2012) Impfquoten bei der Schuleingangsuntersuchung in Deutschland 2010. Epidemiol Bull 16:135-139

8. Robert Koch-Institut (2008) Pertussis: Zum Vergleich von Grundimmuniserung und Auffrischimpfungen in den alten und neuen Bundesländern: Daten aus dem Kinder und Jugendgesundheitssurvey (KiGGS). Epidemiol Bull 27:213-214

9. STIKO (2006) Empfehlung der Ständigen Impfkommission (STIKO) am Robert Koch-Institut zur Pertussis-Schutzimpfung. Stand: Januar 2006. Epidemiol Bull 3:21-23

10. Robert Koch-Institut (2010) Zur Situation bei ausgewählten Infektionskrankheiten in Deutschland. Keuchhusten-Erkrankungen in den neuen Bundesländern seit 2007. Epidemiol Bull 47:463-471

11. STIKO (2009) Empfehlungen der Ständigen Impfkommission (STIKO) am Robert Koch-Institut. Stand: Juli 2009. Epidemiol Bull 30:279-298

12. Cherry JD (2012) Why do pertussis vaccines fail? Pediatrics 129(5):968-970

13. Robert Koch-Institut (2010) Pertussis (Keuchhusten) RKI-Ratgeber für Ärzte. Epidemiol Bull. http:// www.rki.de/DE/Content/Infekt/EpidBull/Merkblaetter/Ratgeber_Pertussis.html (Zugegriffen: 04.02.2013)

14. Guiso N, Berbers G, Fry NK et al (2011) What to do and what not to do in serological diagnosis of pertussis: recommendations from EU reference laboratories. Eur J Clin Microbiol Infect Dis 30(3):307-312

15. Riffelmann $M$, Littmann $M$, Hellenbrand $W$ et al (2008) Pertussis - nicht nur eine Kinderkrankheit. Dtsch Arztebl 105(37):623-628

16. Cherry JD, Seaton BL (2012) Patterns of Bordetella parapertussis respiratory illnesses: 2008-2010. Clin Infect Dis 54(4):534-537

17. Gesetz zur Neuordnung seuchenrechtlicher Vorschriften (Seuchenrechts-Neuordnungsgesetz SeuchRNeuG) vom 20. Juli 2000. Artikel 1 Gesetz zur Verhütung und Bekämpfung von Infektionskrankheiten beim Menschen (Infektionsschutzgesetz - IfSG) Bundesgesetzblatt (BGBI). I S. 1045. 2000. $20 \mathrm{Jul} 2000.2$
18. WHO Europe (2012) Eliminating measles and rubella. Framework for the verification process in the WHO European Region. http://www.euro.who. int/_data/assets/pdf_file/0005/156776/e96153Eng.pdf (Zugegriffen: 04.02.2013)

19. Siedler A, Arndt U (2010) Impact of the routine varicella vaccination programme on varicella epidemiology in Germany. Euro Surveill 15(13):pii=19530. http://www.eurosurveillance. org/ViewArticle.aspx?Articleld=19530

20. Spackova M, Muehlen M, Siedler A (2010) Complications of varicella after implementation of routine childhood varicella vaccination in Germany. Pediatr Infect Dis J 29(9):884-886 